\title{
Neuropsychological performance after carotid endarterectomy
}

\author{
Lenka Krámská ${ }^{1,2, *}$, Martin Kovár̆ ${ }^{2}$, Lucia Hrešková ${ }^{1}$, Martin Jerie ${ }^{2,3}$ \\ ${ }^{1}$ Department of Clinical Psychology, Na Homolce Hospital, 15000 Prague, Czech Republic \\ ${ }^{2}$ Department of Neurology, Na Homolce Hospital, 15000 Prague, Czech Republic \\ ${ }^{3}$ Charles University, First Faculty of Medicine, 12000 Prague, Czech Republic \\ "Correspondence: lenka.kramska@homolka.cz (Lenka Krámská) \\ DOI:10.31083/j.jin2101036
}

This is an open access article under the CC BY 4.0 license (https://creativecommons.org/licenses/by/4.0/).

Submitted: 29 July 2021 Revised: 15 September 2021 Accepted: 18 September 2021 Published: 28 January 2022

Background: Internal carotid endarterectomy (CEA) is a method of stroke prevention in patients with severe internal carotid artery (ICA) stenosis. Patients with significant carotid stenosis tend to have lower cognitive performance than those without significant stenosis. This is believed to be due to hypoperfusion or ongoing microembolization to the brain. Methods: We evaluated 60 patients with at least $70 \%$ ICA stenosis with the RBANS test (Repeatable Battery for the Assessment of Neuropsychological Status, Czech research version), preoperatively and one month after endarterectomy. Results: Neuropsychological follow-up was completed by $\mathrm{N}=57$ patients one month after the procedure. At the group level, there was a significant improvement in Language, Attention, Delayed Memory and Total Scale Index Scores $(p<0.05)$. Conclusions: CEA not only decreases the long-term risk of ischemic stroke, but may also improve cognitive performance. In a small percentage of cases, there is higher risk of embolic stroke and silent microembolization due to surgery, which may have a negative impact on cognitive function. However, we did not detect any cognitive impairment after CEA in our patients.

\section{Keywords}

Carotid stenosis; Cognitive performance; Endarterectomy; RBANS

\section{Introduction}

Atherosclerotic carotid disease may lead to transient ischemic attack (TIA) and ischemic stroke. Carotid endarterectomy (CEA) has recently been supported as a treatment option and secondary prevention of brain ischemia in patients with symptomatic stenosis of the internal carotid artery (ICA) [1]. The fact that high-grade carotid stenosis negatively influences cognition is supported by recent studies [2]. Patients with high-grade stenosis of ICA recommended for CEA had lower cerebrovascular reactivity on the stenotic side, and decreased test scores evaluating neuropsychological performance of the ipsilateral hemisphere [3]. Prior research however has not conclusively established a relationship between neuropsychological performance and stenosis intervention [4].

"Cognitive function" is a term that describes a wide range of psychological processes such as memory, attention, language and mood, and allows one to successfully interact with the surrounding world. Deterioration in cognitive function is considered multifactorial and moreover significantly corre- lates with age, and also has a direct impact on everyday functioning and quality of life $[5,6]$. Typical vascular risk factors (diabetes, dyslipidemia, hypertension and smoking) are also risk factors for the development of carotid stenosis and dementia. Carotid stenosis is usually considered a direct cause of impaired cognitive functioning, which may be an indicator of generalized atherosclerosis. Cognitive deficit seen in patients with symptomatic stenosis may be easily explained by the presence of ischemic brain injury $[5,7]$.

Asymptomatic stenosis is defined as stenosis of the ICA without incidence of TIA, retinal ischemia, or ipsilateral stroke. Research findings demonstrate the clinical significance of carotid atherosclerosis and asymptomatic carotid artery stenosis beyond the risk of stroke, by elevating the incidence of cognitive impairment and dementia. However, this is relevant not only in patients diagnosed with vascular dementia, but also in Alzheimer disease, due to the occurrence of analogous risk factors and pathophysiological findings with vascular dementia [8]. Progressive cognitive decline in vascular disease may be even more prevalent than stroke, but it has not been widely acknowledged and therefore must be addressed accordingly [5].

Anatomical changes caused by decreased blood flow may also play an important role in the development of cognitive deficit. Impaired cerebral hemodynamic has been connected to ipsilateral cortical thinning and, following surgical revascularization, restored cortical thickness $[9,10]$. It has been suggested that surgery for carotid stenosis in patients with significant cognitive impairment may be beneficial. Hypoperfusion may lead to deficits in brain regions previously impaired but can be restored in otherwise healthy tissue [11].

Assessment of cognitive performance is often problematic, as patients can exhibit difficulties in concentration [6]. Individuals with carotid stenosis often suffer from deficits in other cognitive domains, especially attention, memory and executive function, which are influenced by frontal cerebral blood flow (CBF). Neurocognitive performance may be ameliorated after carotid revascularization, and these positive changes have been shown to correlate with improved cerebral hemodynamics $[12,13]$. 
Table 1. Procedural characteristics.

\begin{tabular}{|c|c|c|c|c|c|c|}
\hline \multicolumn{7}{|c|}{ Characteristic $N=60$} \\
\hline Side of ICA stenosis & Left & & $26(43 \%)$ & Right & & $57 \%)$ \\
\hline General anesthesia & \multicolumn{6}{|c|}{$60(100 \%)$} \\
\hline Cross-clamp to shunting time (s) & Median & 96 & Range & $51-180$ & IQR & $75-120$ \\
\hline Shunt extraction time (s) & Median & 60 & Range & $30-140$ & IQR & $55-84.5$ \\
\hline Early reoperation & \multicolumn{6}{|c|}{$2(3 \%)$} \\
\hline
\end{tabular}

$\mathrm{IQR}$, interquartile range; $\mathrm{N}$, number; s, seconds.

The present neuropsychological study aimed to examine the relationship between preoperative individual characteristics and changes in cognitive function in patients with highgrade ICA stenosis one month after CEA. For the purposes of this study, we used comprehensive neuropsychological assessment to evaluate primary cognitive domains.

\section{Materials and methods}

Between October 2016 and January 2020, patients treated by endarterectomy at our tertiary comprehensive stroke center that fulfilled the inclusion criteria were subjected to neuropsychological evaluation. The inclusion criteria consisted of: (1) At least 70\% ICA stenosis diagnosed by both ultrasound and angiography of any kind. (2) Pre-operative modified Rankin scale score of 0-2. (3) Temporal bone thickness allowing transcranial insonation. (4) Completed preoperative neuropsychological examinations. (5) The provision of signed, informed consent.

\subsection{Neurological and vascular examination}

The degree of carotid stenosis was evaluated by duplex ultrasound and confirmed by computed tomography (CT) or magnetic resonance imaging (MRI) angiography. Ultrasound examination was performed by one of three experienced neurosonologists. The North American Symptomatic Carotid Endarterectomy Trial (NASCET) criteria were applied and signs of severe hemodynamic dysfunction were requisite; only patients with stenosis $\geq 70 \%$ were included in the study. All patients underwent a neurological examination preoperatively, two hours postoperatively, prior to discharge and one month postoperatively.

\subsection{Carotid surgery}

All patients underwent general anesthesia and shunting. Heparin 150 units $/ \mathrm{kg}$ was administered 3 minutes before carotid cross-clamping and protamine sulfate was given after vascular suture. Ischemia cross-clamp time, when documented, never exceeded 180 seconds. The median value of cross-clamp to shunting was 96 seconds (IQR 75-120 s) and time for shunt extraction had a median value of $60 \mathrm{~s}$ (IQR 55-85 s). However, cross-clamp time measurements were not available in 16 (27\%) cases. Throughout the operation and 1-2 days postoperatively, all patients underwent invasive blood pressure (BP) monitoring and active BP control by an experienced anesthesiologist/intensivist. The length of hospital stay was typically five days. Two (3\%) patients required early reoperation for hematoma, with no sequelae. In 1 patient, contralateral high-grade carotid stenosis was surgically treated 6 weeks after index endarterectomy under regional anesthesia. This patient was neuropsychologically evaluated before the first operation and then one month after the second operation; no complications were noted. Detailed procedural characteristics are summarized in Table 1.

\subsection{Neuropsychological examination}

All patients were examined by a registered clinical psychologist (L.K.) preoperatively, 1 month and 1 year (not included in the present study) after CEA. Neuropsychological examination included a detailed diagnostic interview, medical history, and administration of the Czech research version of RBANS (Repeatable Battery for the Assessment of Neuropsychological Status). The total duration of the examination was 90 minutes on average, and patients were informed about their test results. The day prior to surgery, RBANS version A was administered, and one month after surgery RBANS version $B$. The test has 4 parallel versions, making it suitable for repeated examinations as the practice effect is minimized. Using RBANS, six index scores can be determined: Total Score, Immediate Memory, Visuospatial/Constructional, Language, Attention, and Delayed Memory. The mean of the Index Scores is 100 and standard deviation 15. All test scores were corrected for age and education according to normative data.

\subsection{Statistical analyses}

Values are shown as mean and standard deviation (SD) for continuous variables and as the number (percent) of subjects for categorical variables. A paired $t$-test was used for the evaluation of differences in cognitive performance before and after surgery. Differences in scores were assessed by the Wilcoxon paired test. Differences in cognitive performance were calculated by subtracting the baseline values from values obtained on neuropsychological tests 1 month after CEA. Statistica version 13.0 software (StatSoft, Inc., OK, USA) was used for statistical analyses.

\section{Results}

Sixty patients ( 17 female) were included in the study, with a mean age of 67.9 years $(\mathrm{SD}=8.7$, range $=47-83)$. The average education obtained was 13.2 years $(\mathrm{SD}=3.33)$. Twentytwo (37\%) patients were deemed symptomatic before inclusion, having had a transient ischemic attack, stroke or (tran- 
sient) retinal ischemia on the index side. Fifty-one (85\%) subjects had hypertension, 49 (82\%) were current or past smokers, 22 (37\%) had diabetes and 21 (35\%) coronary artery disease. Detailed characteristics of patients are presented in Table 2.

Table 2. Preoperative patient characteristics, personal history.

\begin{tabular}{lcc}
\hline Characteristics & $\mathrm{N}=60$ & (\%) \\
\hline Female sex & 17 & 28 \\
Arterial hypertension & 51 & 85 \\
Diabetes mellitus & 22 & 37 \\
Coronary artery disease & 21 & 35 \\
Smoking current + past & 49 & 82 \\
Smoking current & 28 & 47 \\
Symptomatic carotid stenosis & 22 & 37 \\
Stroke/TIA in patients taking ASA (before CEA) & 9 & 15 \\
\hline
\end{tabular}

$\mathrm{N}$, number; TIA, transient ischemic attack; ASA, acetylsalicylic acid; CEA, carotid endarterectomy.

Fifty-four (90\%) patients were taking acetylsalicylic acid (ASA) at the time of operation, 7 subjects were on clopidogrel or dual treatment with ASA, 5 had anticoagulation with temporary discontinuation or reduced dose. Thirty-four (57\%) patients underwent surgery on the right side, 25 patients on the left and one bilaterally, six weeks apart. Two (3\%) subjects were left-handed, the remainder were right-handed. The post-operative neuropsychological follow-up was completed by 57 (95\%) patients 1 month after the procedure. Three patients were not willing to undergo follow-up testing; they showed no neurological complications at one-month followup.

Interestingly, we did not detect any instances of postoperative stroke or transient ischemic attack (TIA) within 1 month of CEA. However, postoperative MRI or CT were not performed, we therefore cannot exclude subclinical embolic microinfarctions. Using duplex sonography, we observed no severe restenosis or occlusion 1 month postoperatively, with 3 patients showing asymptomatic restenosis later. At the group level, there was a significant improvement in Language, Attention, Delayed Memory and Total Scale Index Score $(p<0.05)$. Detailed pre- and post-surgical neuropsychological test results are summarized in Table 3.

\section{Discussion}

In the present study, we found characteristic alterations in Attention, Immediate Memory and Total Scale Index Scores $(<1$ SD below average) at baseline assessment, before CEA. We further observed significant improvement in Language, Attention, Delayed Memory and Total Index Scores postoperatively. Increased postoperative scores may be partly explained by the practice effect, however, to minimize this phenomenon we administered parallel versions of neuropsychological batteries (RBANS A and B). According to our results,
CEA may improve cognitive performance in patients with significant carotid stenosis.

Many studies have investigated the neurocognitive outcome in patients treated with CEA, however, the results have been inconsistent as they demonstrated either improvements or no functional changes post-surgery [14]. In a study by Wang et al. [15] increased cerebral perfusion was observed in a group of patients. Their study implies that asymptomatic status had no significant impact on cognitive performance, which suggests that CEA may improve cerebral perfusion in both asymptomatic and symptomatic patients. Regarding cognitive results, total MMSE (Mini-Mental State Examination) scores were similar in pre- and post-operative groups, but the post-operative group performed better on the "recall" subtest compared to baseline. In contrast, the total MoCA (Montreal Cognitive Assessment) score improved significantly after CEA, as well as subtests including "attention", "naming”, "visuo-executive", and "abstraction”. Interestingly however, no improvement was found on the MoCA "recall" subtest. On MoCA subtests, "orientation" and "verbal fluency" demonstrated the highest pre-procedural scores, with "visuo-executive" and "recall" demonstrating the lowest. After CEA, MoCA subtests of "abstraction", "attention", and "naming" showed more improvement than other subtests. The "recall" subtest also had the lowest MMSE score. Differences in cognitive profile pre- and post-intervention were more apparent on MoCA than MMSE. These results show the need for more detailed and sensitive tests for neuropsychological assessment.

Watanabe et al. [16] reported improved executive function in patients that underwent CEA. Additionally, in an earlier study, Watanabe et al. [17] reported that CEA may lead to improved cognitive performance in patients with significant carotid stenosis. A sub-analysis confirmed that only the memory score increased after CEA. Improved cognitive function was detected in patients with severe $(80 \%)$ or bilateral carotid stenosis. This may due to increased cerebral blood flow in these patients postoperatively.

Schröder et al. [8] reported no significant relationship between measures of perfusion and cognitive performance, and no significant changes in cognitive test scores after revascularization. However, there was a trend towards increased cognitive processing speed as evaluated by the Trail Making Test part A.

There is evidence that mild cognitive impairment may be present in $27-31 \%$ of patients after CEA. This may be due to decreased brain perfusion and embolism, or other individual factors [18-20]. Some studies have found an association between high-grade $(\geq 75 \%)$ stenosis of the left internal carotid artery and cognitive impairment, but also cognitive decline during follow-up, with no correlation observed in patients with right-sided stenosis $[5,17]$.

A study by Ge et al. [7] found that $33 \%$ of patients showed decreases in MMSE and MOCA scores at 6 and 24 hours postoperatively, compared with baseline. This functional 
Table 3. Neuropsychological performance before and one month after CEA.

\begin{tabular}{lccc}
\hline \multirow{2}{*}{ RBANS Index Score } & Before surgery & One month after surgery & \multirow{2}{*}{-value } \\
\cline { 2 - 3 } & RBANS A & RBANS B & \\
\cline { 2 - 3 } & M (SD) & M (SD) & 0.955 \\
\hline Immediate Memory & $85.23(15.2)$ & $85.14(15.28)$ & 0.287 \\
Visuospatial/Constructional Index Score & $93.04(15.48)$ & $94.79(18.56)$ & $<\mathbf{0 . 0 0 1}$ \\
Language Index Score & $96.02(11.56)$ & $104.05(15.14)$ & $\mathbf{0 . 0 2 4}$ \\
Attention Index Score & $78.54(14.41)$ & $81.98(14.04)$ & $\mathbf{0 . 0 0 8}$ \\
Delayed Memory Index Score & $91.23(18.04)$ & $95.84(18.43)$ & $<\mathbf{0 . 0 0 1}$ \\
Total Scale Index Score & $85.26(13.89)$ & $89.83(16.02)$ & \\
\hline
\end{tabular}

Significant differences $(p<0.05)$ in both subgroups are in bold.

decline may be due to oxidative stress, residual anesthetic effects, cerebral microemboli intra- and post-operatively, or prolonged global cerebral ischemia during carotid crossclamping.

In a review by Wang et al. [5], the following potential reasons for cognitive impairment after CEA were proposed: (1) A transient decrease in perfusion during carotid crossclamping may cause neural damage. (2) A large percentage of patients demonstrated evidence of gaseous and particulate emboli intra-operatively and post-CEA. Silent embolization occurred in approximately $9 \%$ of patients. (3) General anesthesia, diabetes mellitus, cerebral hyperperfusion after CEA, and the absence of both posterior communicating arteries are all considered risk factors for cognitive dysfunction after CEA. Cognitive function may also be negatively influenced by preclinical Alzheimer disease.

The perioperative frequency of stroke after CEA is low, however "silent" microinfarctions confirmed by MRI are frequent and have been correlated with postoperative neurocognitive impairment [21].

Older age is associated with a higher occurrence of structural brain pathology, such as silent ischemic infarcts and white matter lesions. These may be considered as determinants of cognitive impairment and are not reversable through revascularization [22].

A wide variety of factors can interfere with cerebral perfusion and cognitive function, such as age, white matter lesions, hypertension and diabetes, which may explain the conflicting results reported in previous studies.

Stroke prevention is still the main aim of surgery in patients with carotid stenosis, however, improvement in cognitive functionning may be of importance, especially for individuals that are commonly required to perform intellectuallydemanding tasks. It would be logical to include detailed neuropsychological evaluation as an outcome measure in research studies evaluating treatment approaches for carotid artery occlusive disease $[2,7,14]$.

The major strengths of the present study include prospective evaluation, the estimate of cognitive domains with two versions of a neuropsychological battery and the one-month period between neuropsychological testing to lower the practice effect. The generalizability of our findings is limited by the selective inclusion criteria to patients with history of TIA and unilateral high-grade ICA stenosis. This, however, allowed us to avoid the potentially confounding impact of poststroke recovery or plasticity, and to investigate changes primarily influenced by revascularization.

The present study has some limitations. First, the number of participants was relatively small, and therefore we did not include other factors such as gender, age and laterality in the statistical analyses. Second, the study was conducted at a single stroke center. The neuropsychological results could be improved by the use of more sensitive measures of executive and memory function in a larger cohort of patients. Third, although we aimed to include consecutive patients, some subjects were not involved in the study due to insufficient time to complete the neuropsychological tests preoperatively. This may have excluded some symptomatic patients indicated urgently for intervention. Finally, we report results at 1 month after intervention, while some studies have tested patients after a longer period. However, our preliminary analyses of outcomes 1 year after intervention show the same trend.

\section{Conclusions}

Our findings may provide insight into the controversial impact of stenosis intervention on cognitive function. We acknowledge that the heterogeneity of patients with respect to preoperative characteristics and the absence of analysis related to the hemodynamic influence of ICA stenosis may explain the contradictory results between studies [13]. Our results may prove insightful with regard to cognitive performance before CEA and 1 month after the procedure. Despite impaired cognitive performance before surgery (Attention, Immediate Memory and Total Score domains), we observed significant improvement in several domains: Language, Attention, Delayed Memory and Total Score. A larger sample would be needed for more detailed analyses considering premorbid intellectual performance, cognitive impairment, age, gender, laterality of intervention, stroke and other neurological and medical complications before the intervention. More sensitive neuropsychological tests, which are able to localize specific cortical functional zones, should also be included in future studies. New imaging techniques could possibly broaden our understanding of the relationship between cognitive function and blood flow [5]. 


\section{Abbreviations}

BP, blood pressure; CBF, cerebral blood flow; CEA, Internal carotid endarterectomy; CT, computed tomography; ICA, internal carotid artery; MoCA, Montreal Cognitive Assessment; MMSE, Mini-Mental State Examination; MRI, magnetic resonance imaging; RBANS, Repeatable Battery for the Assessment of Neuropsychological Status; SD, standard deviation; TIA, transient ischemic attack.

\section{Author contributions}

LK prepared the conception and design of the study, participated in the acquisition and analysis of data. LK wrote the original draft of the manuscript and critically revised the manuscript. MK, LH and MJ participated in the acquisition and analysis of data, critically revised and edited the manuscript. All the authors meet the standard criteria of authorship based on recommendations of the international committee of medical journal editors.

\section{Ethics approval and consent to participate}

All data collection, storage and processing were done in compliance with the Helsinki Declaration. All patients provided signed, informed consent and the study number IG 1.7.2015/10 was approved by the ethics committee of $\mathrm{Na} \mathrm{Ho-}$ molce Hospital in Prague.

\section{Acknowledgment}

We thank Aaron Rulseh for English editing.

\section{Funding}

The study was supported by the Ministry of Health of the Czech Republic, grant MH CZ and DRO NHH, IG 161501.

\section{Conflict of interest}

The authors declare no conflicts of interest.

\section{References}

[1] Abbott AL, Paraskevas KI, Kakkos SK, Golledge J, Eckstein H, Diaz-Sandoval LJ, et al. Systematic Review of Guidelines for the Management of Asymptomatic and Symptomatic Carotid Stenosis. Stroke. 2016; 46: 3288-3301.

[2] Kougias P, Collins R, Pastorek N, Sharath S, Barshes NR, McCulloch $\mathrm{K}$, et al. Comparison of domain-specific cognitive function after carotid endarterectomy and stenting. Journal of Vascular Surgery. 2015; 62: 355-361.

[3] Hitchner E, Baughman BD, Soman S, Long B, Rosen A, Zhou W. Microembolization is associated with transient cognitive decline in patients undergoing carotid interventions. Journal of Vascular Surgery. 2016; 64: 1719-1725.

[4] Lattanzi S, Carbonari L, Pagliariccio G, Cagnetti C, Luzzi S, Bartolini M, et al. Predictors of cognitive functioning after carotid revascularization. Journal of the Neurological Sciences. 2019; 405: 116435.

[5] Wang T, Mei B, Zhang J. Atherosclerotic carotid stenosis and cognitive function. Clinical Neurology and Neurosurgery. 2017; 146: 64-70.
[6] Kalimeris K, Kouni S, Kostopanagiotou G, Nomikos T, Fragopoulou E, Kakisis J, et al. Cognitive function and oxidative stress after carotid endarterectomy: comparison of propofol to sevoflurane anesthesia. Journal of Cardiothoracic and Vascular Anesthesia. 2014; 27: 1246-1252.

[7] Ge Y, Li Q, Nie Y, Gao J, Luo K, Fang X, et al. Dexmedetomidine improves cognition after carotid endarterectomy by inhibiting cerebral inflammation and enhancing brain-derived neurotrophic factor expression. Journal of International Medical Research. 2019; 47: 2471-2482.

[8] Schröder J, Heinze M, Günther M, Cheng B, Nickel A, Schröder $\mathrm{T}$, et al. Dynamics of brain perfusion and cognitive performance in revascularization of carotid artery stenosis. NeuroImage: Clinical. 2019; 22: 101779.

[9] Yoshida J, Yamashita F, Sasaki M, Yoshioka K, Fujiwara S, Kobayashi M, et al. Adverse effects of pre-existing cerebral small vessel disease on cognitive improvement after carotid endarterectomy. International Journal of Stroke. 2020; 15: 657-665.

[10] Marshall RS, Asllani I, Pavol MA, Cheung YK, Lazar RM. Altered cerebral hemodyamics and cortical thinning in asymptomatic carotid artery stenosis. PLoS ONE. 2017; 12: e0189727.

[11] Heller S, Hines G. Carotid Stenosis and Impaired Cognition: the Effect of Intervention. Cardiology in Review. 2017; 25: 211-214.

[12] Lattanzi S, Carbonari L, Pagliariccio G, Bartolini M, Cagnetti C, Viticchi G, et al. Neurocognitive functioning and cerebrovascular reactivity after carotid endarterectomy. Neurology. 2019; 90: 307315.

[13] Plessers M, Van Herzeele I, Vermassen F, Vingerhoets G. Neurocognitive functioning after carotid revascularization: a systematic review. Cerebrovascular Diseases Extra. 2014; 4: 132-148.

[14] Saito H, Ogasawara K, Nishimoto H, Yoshioka Y, Murakami $\mathrm{T}$, Fujiwara S, et al. Postoperative changes in cerebral metabolites associated with cognitive improvement and impairment after carotid endarterectomy: a 3T proton MR spectroscopy study. American Journal of Neuroradiology. 2013; 34: 976-982.

[15] Wang Q, Zhou M, Zhou Y, Ji J, Raithel D, Qiao T. Effects of Carotid Endarterectomy on Cerebral Reperfusion and Cognitive Function in Patients with High Grade Carotid Stenosis: a Perfusion Weighted Magnetic Resonance Imaging Study. European Journal of Vascular and Endovascular Surgery. 2015; 50: 5-12.

[16] Watanabe J, Ogata T, Higashi T, Inoue T. Cognitive Change 1 Year after CEA or CAS Compared with Medication. Journal of Stroke and Cerebrovascular Diseases. 2017; 26: 1297-1305.

[17] Watanabe J, Ogata T, Hamada O, Nonaka M, Abe H, Higashi $\mathrm{T}$, et al. Improvement of cognitive function after carotid endarterectomy-a new strategy for the evaluation of cognitive function. Journal of Stroke and Cerebrovascular Diseases. 2015; 23: 1332-1336.

[18] Berman L, Pietrzak RH, Mayes L. Neurocognitive changes after carotid revascularization: a review of the current literature. Journal of Psychosomatic Research. 2008; 63: 599-612.

[19] De Rango P, Caso V, Leys D, Paciaroni M, Lenti M, Cao P. The role of carotid artery stenting and carotid endarterectomy in cognitive performance: a systematic review. Stroke. 2008; 39: $3116-$ 3127.

[20] Heyer EJ, DeLaPaz R, Halazun HJ, Rampersad A, Sciacca R, Zurica $\mathrm{J}$, et al. Neuropsychological dysfunction in the absence of structural evidence for cerebral ischemia after uncomplicated carotid endarterectomy. Neurosurgery. 2006; 58: 474-480.

[21] Sridharan ND, Robinson D, Thirumala P, Arak A, Olafiranye O, Tzeng E, et al. Rationale and Design for the Remote Ischemic Preconditioning for Carotid Endarterectomy Trial. Annals of Vascular Surgery. 2019; 60: 246-253.

[22] Habes M, Erus G, Toledo JB, Zhang T, Bryan N, Launer LJ, et al. White matter hyperintensities and imaging patterns of brain ageing in the general population. Brain. 2016; 139: 1164-1179. 